\title{
Retinal nerve fiber layer thickness in children with primary congenital glaucoma measured by spectral domain optical coherence tomography
}

\author{
Lucía Perucho-González, MD, PhD, ${ }^{\mathrm{a}, \mathrm{c}}$ José María Martínez de la Casa, MD, PhD, a,b,c,d \\ Federico Sáenz-Francés, $\mathrm{MD}, \mathbf{P h D},{ }^{\mathrm{a}, \mathrm{c}}$ Laura Morales-Fernandez, $\mathrm{MD}, \mathrm{PhD},{ }^{\mathrm{a}, \mathrm{c}}$ \\ Carmen Dora Méndez-Hernández, MD, PhD, ${ }^{\mathrm{a}, \mathrm{b}, \mathrm{c}, \mathrm{d}}$ Rubén Sánchez-Jean, ${ }^{\mathrm{b}, \mathrm{c}, \mathrm{d}}$ and \\ Julián García-Feijoó, MD, PhD ${ }^{\mathrm{a}, \mathrm{b}, \mathrm{c}, \mathrm{d}}$
}

PURPOSE

To evaluate retinal nerve fiber layer (RNFL) thickness using spectral domain optical coherence tomography (SD-OCT) in a population of children diagnosed with primary congenital glaucoma (PCG).

METHODS

In this cross-sectional study, 59 eyes of 59 children diagnosed with PCG and 87 eyes of 87 healthy children were evaluated by SD-OCT to measure the RNFL. The global average peripapillary RNFL thickness and sectional RNFL thickness were evaluated in both groups. Differences in global average and sectional thickness were analyzed.

RESULTS Mean age in the PCG group was $9.61 \pm 3.23$ years; in the control group, $8.47 \pm 2.99$ years $(P=0.0516)$. There were statistically significant differences $(P<0.007)$ in all sectors between both groups.

CONCLUSIONS SD-OCT is a promising tool for evaluating the eyes of children diagnosed with PCG. Future research should examine the test-retest variability of SD-OCT parameters and their ability to diagnose progression in these children. (J AAPOS 2019; $\mathbf{0}: 1 . e 1-4)$

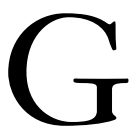
laucoma in children is characterized by the presence of elevated intraocular pressure (IOP) and characteristic optic disk cupping. Currently the most accepted methods for establishing the glaucomarelated optic nerve changes in children are optic nerve head examination and stereo photography. Some studies have reported differences in the retinal nerve fiber layer (RNFL) thickness between normal and glaucomatous eyes in children evaluated with optical coherence tomography (OCT) using both time domain ${ }^{1}$ and spectral domain $(\mathrm{SD}-\mathrm{OCT})^{2}$ systems. The current study investigated the role of ST-OCT in diagnosing glaucoma in children.

\section{Subjects and Methods}

This observational cross-sectional study included healthy volunteers and patients diagnosed with primary congenital glaucoma

\footnotetext{
Author affiliations: ${ }^{a}$ Ophthalmology Service, Institute of Sanitary Research of the San Carlos Clinical Hospital, Madrid, Spain; ${ }^{b}$ Ocular Patbology, National Net OFTARED, Institute of Health Carlos III, University Hospital, Madrid, Spain; ${ }^{c}$ Glaucoma Department, Institution of Sanitary Research of the San Carlos Clinical Hospital, Madrid, Spain; ${ }^{d}$ Universidad Complutense, Madrid, Spain

Submitted August 17, 2016.

Revision accepted March 3, 2017

Correspondence: Lucía Perucho-González, Ocular Pathology National Net OFTARED of the Institute of Health Carlos III University Hospital, Profesor Martín Lagos Street, 28040 Madrid, Spain (email: luciaperuchogonz@gmail.com).

Copyright (C) 2019, American Association for Pediatric Ophthalmology and Strabismus. Published by Elsevier Inc. All rights reserved.

1091-8531/\$36.00

https://doi.org/10.1016/j.jaapos.2017.03.023
}

(PCG) treated in the glaucoma department of San Carlos Clinical Hospital, Madrid, a tertiary eye care center. The study was approved by the San Carlos University Hospital Institutional Review Board and complied with the guidelines of the Declaration of Helsinki. Written informed consent was obtained from the legal guardians of minors before enrollment, with the assent of children $>12$ years of age.

Children with known PCG-IOP of $>22 \mathrm{~mm} \mathrm{Hg}$ at diagnosis together with clinical evidence of glaucoma, such as enlarged corneal diameter, Haab's striae, glaucomatous optic disk head appearance, and glaucomatous visual fields (when available) were enrolled. Only PCG patients with IOP of $<21 \mathrm{~mm} \mathrm{Hg}$ after either medical therapy or surgery performed at least 6 months earlier and patients able to fixate on a target were included. Patients with other childhood glaucoma, presence of corneal edema precluding optical coherence tomography, nystagmus, uveitis, any concomitant retinal disease, or significant media opacity, such as cataract or pseudophakia, were excluded.

A total of 87 healthy volunteers were recruited from children referred for refraction who had normal examinations, children accompanying adult patients, and children of staff members who agreed to participate in the study. They had a normal eye examination, including a normal optic disk, IOP of $<21 \mathrm{~mm} \mathrm{Hg}$, and no history of ocular surgery or trauma. Only one eye was randomly chosen in each participant unless only one eye met inclusion criteria (www.randomization.com).

All subjects underwent full ophthalmic examination, including dilated fundus examination, in one session. IOP was measured by 
Perkins handheld applanation tonometer (Haag-Streit, Koniz, Switzerland) when possible. Icare rebound tonometry (TA01I; Icare, Finland) was used if corneal irregularities were present or when cooperation was poor. All OCT scans were obtained by the same trained ophthalmologist.

SD-OCT was used to obtain the RNFL (Spectralis; Heidelberg Engineering GmbH, Heidelberg, Germany; software version 5.4) thickness maps. A full RNFL circle scan containing $768 \mathrm{~A}$-scans along a peripapillary circle of $360^{\circ}$ was performed. The Spectralis software divides this circle into six regions: temporal quadrant thickness $\left(90^{\circ}\right.$ sector), superotemporal quadrant thickness $\left(45^{\circ}\right.$ sector), superonasal quadrant thickness ( $45^{\circ}$ sector), nasal quadrant thickness $\left(90^{\circ}\right.$ sector), inferonasal quadrant thickness $\left(4^{\circ}\right.$ sector), inferotemporal quadrant thickness $\left(45^{\circ}\right.$ sector), and average thickness (average of all 6 sectors or $360^{\circ}$ ), provided as a number in the center.

Scans were included for analysis if the quality score was $>20 \mathrm{~dB}$. Three images were automatically obtained for each eye during the same session. If none of the three images met the quality criteria the eye was excluded from the analysis.

\section{Statistical Analysis}

All statistical tests were performed using the SPSS package version 18.0 for Windows (SPSS Inc, Chicago, IL). The Kolmogorov-Smirnov test was used to check for a normal distribution of quantitative data. Quantitative variables were expressed as their corresponding means and standard deviations. Medians and interquartile ranges were used to describe variables showing a non-normal distribution. Correlation between different treatments in PCG and RNFL thickness was assessed using the Pearson correlation coefficient. Bonferroni correction of the $P$ value was performed for multiple comparisons and the level of significance was set at $P$ value of $<0.007$. The diagnostic capacity of each variable to differentiate between normal and glaucoma eyes was determined by calculating the area under the receiver operating characteristics (ROC) curve (AUC). Differences between the ROC curves were tested to compare AUCs using the Hanley-McNeil method. ${ }^{4}$

\section{Results}

A total of 71 eyes of 71 children diagnosed with PCG and 98 eyes of 98 healthy children were eligible for inclusion. In the PCG group, 5 eyes with poor fixation, 4 eyes with low signal strength index on OCT, and 3 eyes with corneal opacities were excluded from the final analysis. In the control group, 6 eyes with poor fixation and 5 eyes with low signal strength index were excluded. For statistical analysis, 59 eyes of 59 children formed the PCG group; 87 eyes of 87 healthy children, the control group.

In the PCG group, mean age was $9.61 \pm 3.23$ years (range, 3-18); in the control group, $8.47 \pm 2.99$ years (range, 6-18; $P=0.0516$ ). There were no significant differences in terms of sex distribution between groups. In the PCG group, the average age of diagnosis was $23.24 \pm 9.28$ months. Control eyes showed a significantly higher quality score $(29.79 \mathrm{~dB})$ compared with PCG eyes
(25.63 dB; $P<0.0001)$. Clinical measurements of cup:disk ratio were $0.4 \pm 0.1$ in the control group and $0.6 \pm 0.2$ in PGC patients. Peripapillary RNFL measurements obtained in both groups are summarized in Table 1 . There were statistically significant differences $(P<0.007)$ in all sectors between groups, except for the nasal $(P=0.015)$ and inferonasal $(P=0.009)$ sectors. Mean IOP was $19.11 \pm 4.23 \mathrm{~mm} \mathrm{Hg}$ in the PCG group and $13.95 \pm 1.98 \mathrm{~mm} \mathrm{Hg}(P<0.001)$ in the control group.

AUCs for the mean peripapillary RNFL and sectional RNFL thickness measurements are shown in Table 2 and Figure 1. The best AUC parameters were global or average RNFL $(0.831 \mu \mathrm{m})$, temporal superior RNFL $(0.811 \mu \mathrm{m})$, and nasal superior RNFL $(0.770 \mu \mathrm{m})$.

The likelihood ratio for a positive test was 6 , meaning that a positive test is 6 times more likely to come from a PCG patient rather than control. The positive predictive value was 0.8 , and the negative predictive value was 0.6 .

\section{Discussion}

Expert clinical examination of the optic nerve remains the gold standard in diagnosing glaucomatous optic nerve damage in children as well as in adults. Morales and Brown ${ }^{5}$ demonstrated that ultra-short visual field programs that can be completed in $<3$ minutes offer the possibility of testing children successfully. However, they reported that, at $<8$ years of age, variability in individual maturity, concentration, and effort result in cause greater variability in accuracy.

SD-OCT can provide clinicians with objective measurements of RNFL thickness in both adult and pediatric patients. OCT scans have the advantages of being noncontact, rapid, and relatively patient friendly, making them ideally suited for testing children. Our study was a preliminary study to measure the RNFL thicknesses in children with PCG using SD-OCT.

The diagnostic accuracy of SD-OCT RNFL parameters for glaucoma in adults has been investigated extensively. ${ }^{6}$ In general, the parameters with the best diagnostic accuracy are the average peripapillary RNFL thickness and thicknesses in the inferior and superior quadrants. ${ }^{7-12}$ Our results in PCG agree with those reported in glaucoma in adults. We found that the variables featuring the larger AUCs were global or average $(0.831 \mu \mathrm{m})$, temporal superior $(0.811 \mu \mathrm{m})$, nasal superior $(0.770$ microns), temporal inferior $(0.753 \mu \mathrm{m})$, and nasal inferior $(0.748 \mu \mathrm{m})$ RNFL thicknesses.

A well-known phenomenon to take into account is the reversal of optic disk cupping following IOP reduction in congenital and juvenile glaucoma, especially before 1 year of age. ${ }^{13-19}$ This change in optic disk cupping has also been recorded in adults by measuring RNFL thickness, ${ }^{18}$ but the results of these studies seem to be conflicting. For this reason, we decided to exclude PCG patients who had undergone surgery or experienced uncontrolled IOP in the previous 6 months. 
Table 1. Mean values of sectorial RNFL thickness in control group and PCG group

\begin{tabular}{|c|c|c|c|}
\hline $\begin{array}{l}\text { OCT parameter } \\
\text { thickness, } \mu \mathrm{m}\end{array}$ & $\begin{array}{c}\text { Control group, } \\
\text { mean } \pm \mathrm{SD}\end{array}$ & $\begin{array}{l}\text { PCG group, } \\
\text { mean } \pm S D\end{array}$ & $P$ value \\
\hline $\begin{array}{l}\text { RNFL global } \\
\text { RNFL temporal }\end{array}$ & $\begin{array}{r}102.20 \pm 10.54 \\
72.70 \pm 12.17\end{array}$ & $\begin{array}{l}76.83 \pm 23.43 \\
61.66 \pm 19.45\end{array}$ & $\begin{array}{l}0.000 \\
0.001\end{array}$ \\
\hline $\begin{array}{l}\text { RNFL temporal } \\
\text { superior }\end{array}$ & $136.83 \pm 19.49$ & $97.85 \pm 35.01$ & 0.000 \\
\hline $\begin{array}{l}\text { RNFL temporal } \\
\text { inferior }\end{array}$ & $148.09 \pm 23.91$ & $114.25 \pm 45.65$ & 0.000 \\
\hline RNFL nasal & $77.43 \pm 18.72$ & $57.63 \pm 23.37$ & 0.015 \\
\hline $\begin{array}{l}\text { RNFL nasal } \\
\text { superior }\end{array}$ & $111.29 \pm 24.14$ & $76.73 \pm 32.43$ & 0.005 \\
\hline $\begin{array}{l}\text { RNFL nasal } \\
\text { inferior }\end{array}$ & $121.21 \pm 27.63$ & $87.34 \pm 33.99$ & 0.009 \\
\hline
\end{tabular}

$O C T$, optical coherence tomography; $P C G$, primary congenital glaucoma; $R N F L$, retinal nerve fiber layer; $S D$, standard deviation.

${ }^{a}$ Corrected significant difference $(P<0.007)$; $t$ test (Bonferroni post hoc).

Table 2. Results of diagnostic capacity of each variable determined by calculating area under curve (AUC)

\begin{tabular}{lc}
\hline OCT parameter thickness, $\mu \mathrm{m}$ & AUC \\
\hline RNFL global & 0.831 \\
RNFL temporal & 0.666 \\
RNFL temporal superior & 0.811 \\
RNFL temporal inferior & 0.753 \\
RNFL nasal & 0.721 \\
RNFL nasal superior & 0.770 \\
RNFL nasal inferior & 0.748 \\
\hline
\end{tabular}

$O C T$, optical coherence tomography; RNFL, retinal nerve fiber layer.

Yanni and colleauges ${ }^{19}$ reported that the normal mean peripapillary RNFL thickness measured by SD-OCT in 83 healthy children $5-15$ years of age was thicker $(107.6 \pm 1.2 \mu \mathrm{m})$ than mean RNFL thickness in adults.

In central China a school-based cohort study designed to longitudinally observe the development of myopia and other ocular disease among children provided the baseline results in RNFL thickness in a cohort of 7 -year-olds ${ }^{20}$ as well as the RNFL thickness association with refractive error, axial length, and optic disk parameters. ${ }^{21}$

Another recent case-control study of 45 eyes of 37 children who underwent surgery for PCG and 72 eyes of 41 normal children, conducted at a tertiary eye care center in South India, found significant differences in all SD-OCT parameters between both groups. ${ }^{22}$ Our study found that all SD-OCT RNFL thickness measurements were significantly different $(P<0.05)$ in PCG eyes compared with normal healthy controls, suggesting that SD-OCT may be a valuable clinical tool to help monitor glaucoma in the pediatric population. The present study has several limitations. RNFL thickness parameters of the most severe cases are very difficult to obtain, and this could result in a selection bias. An important subgroup of patients of PCG associated with mutations in the CYP1B1 could have been excluded; these patients have more severe PCG, an earlier age of onset, greater corneal alterations, and comparatively more surgical procedures. $^{23,24}$ Our PCG cohort probably consisted of

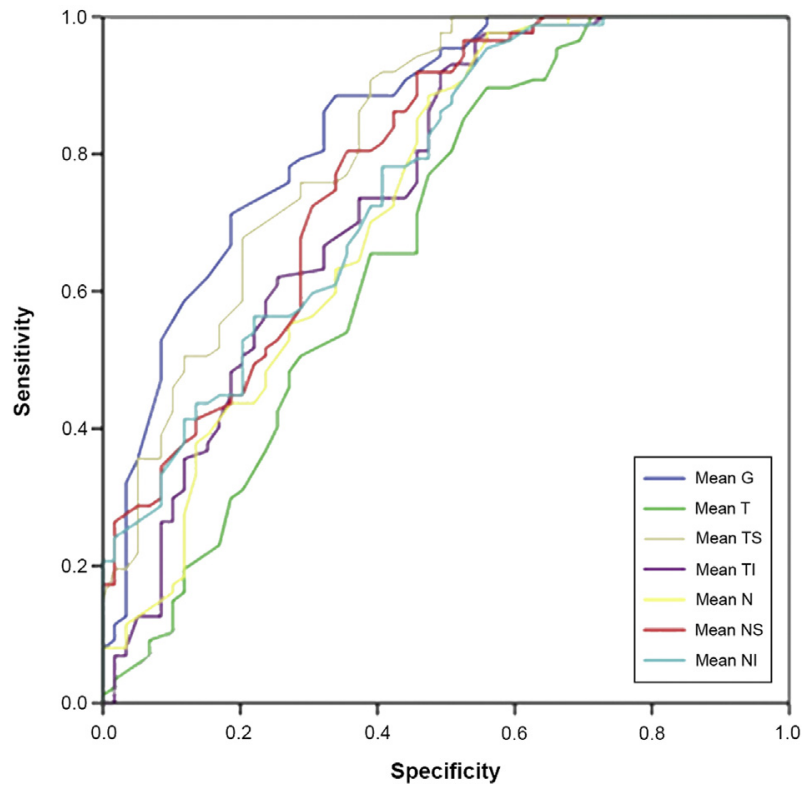

FIG 1. Receiver operating characteristic curves for the variables showing the best discriminant capacity between the two subject groups. AUC: 0.831, RNFL global $(G)$; 0.666, RNFL temporal $(T)$; 0.811, RNFL superotemporal (TS); 0.753, RNFL inferotemporal (TI); 0.721 , RNFL nasal $(M) ; 0.770$, RNFL superonasal (NS); 0.748, RNFL inferonasal $(N /)$.

mild-to-moderate glaucoma cases. The average age at diagnosis in the PCG group was almost 2 years (23.24 \pm 9.28 months); it is possible that most severe cases, which tend to be diagnosed at younger ages, were excluded, resulting in an underestimation of the difference in RNFL thickness between normal and PCG eyes. Also, it is known that axial length affects RNFL measurement in the direction of thinner RNFL and that glaucoma in infancy is associated with ocular enlargement (buphtahalmos), corneal enlargement (usually before three years of age), increased measurements in axial length, and progressive myopia. Thus, we should consider that glaucomatous eyes included probably presented with higher axial length than eyes from control subjects. Future studies should obtain a random distribution of myopic and hyperopic subjects in both normal and glaucomatous subgroups.

Obtaining images of adequate quality using SD-OCT in children can be challenging-more so, perhaps, in children with PCG, who often have low visual acuity or refractive errors. For this reason, in the present study the cut-off of signal-to-noise ratio was fixed at $20 \mathrm{~dB}$; for adults this value tends to be fixed at $40 \mathrm{~dB}$. We found lower quality scores in patients with PCG than in controls, which may affect accuracy of measurement. Future research should also investigate the test-retest variability of SD-OCT parameters and their ability to diagnose progression in these children.

\section{References}

1. Hess DB, Asrani SG, Bhide MG, Enyedi LB, Stinnett SS, Freedman SF. Macular and retinal nerve fiber layer analysis of normal 
and glaucomatous eyes in children using optical coherence tomography. Am J Ophthalmol 2005;139:509-17.

2. Ghasia FF, El-Dairi M, Freedman SF, Rajani A, Asrani S. Reproducibility of spectral-domain optical coherence tomography measurements in adult and pediatric glaucoma. J Glaucoma 2015;24:55-63.

3. Bendschneider D, Tornow RP, Horn FK, et al. Retinal nerve fiber layer thickness in normals measured by spectral domain OCT. J Glaucoma 2010;19:475-82.

4. Hanley JA, McNeil BJ. A method of comparing the areas under receiver operating characteristic curves derived from the same cases. Radiology 1983;148:839-43.

5. Morales J, Brown SM. The feasibility of short automated static perimetry in children. Ophthalmology 2001;108:157-62.

6. Gracitelli CP, Abe RY, Medeiros FA. Spectral-domain optical coherence tomography for glaucoma diagnosis. Open Ophthalmol J 2015; 9:68-77.

7. Park SB, Sung KR, Kang SY, Kim KR, Kook MS. Comparison of glaucoma diagnostic capabilities of cirrus HD and stratus optical coherence tomography. Arch Ophthalmol 2009;127:1603-9.

8. Kim JS, Ishikawa H, Gabriele ML, et al. Retinal nerve fiber layer thickness measurement comparability between time domain optical coherence tomography (OCT) and spectral domain OCT. Invest Ophthalmol Vis Sci 2010;51:896-902.

9. Rao HL, Zangwill LM, Weinreb RN, Sample PA, Alencar LM, Medeiros FA. Comparison of different spectral domain optical coherence tomography scanning areas for glaucoma diagnosis. Ophthalmology 2010;117:1692-9.

10. Leite MT, Rao HL, Zangwill LM, Weinreb RN, Medeiros FA. Comparison of the diagnostic accuracies of the Spectralis, Cirrus, and RTVue optical coherence tomography devices in glaucoma. Ophthalmology 2011;118:1334-9.

11. Leung CK, Lam S, Weinreb RN, et al. Retinal nerve fiber layer imaging with spectral-domain optical coherence tomography. Ophthalmology 2010;117:1684-91.

12. Wang X, Li S, Fu J, et al. Comparative study of retinal nerve fibre layer measurement by RTVue OCT and GDx VCC. Br J Ophthalmol 2011;95:509-13.
13. Meirelles SH, Mathias CR, Bloise RR, et al. Evaluation of the factors associated with the reversal of the disc cupping after surgical treatment of childhood glaucoma. J Glaucoma 2008;17:470-73.

14. Mandal AK, Netland PA. The Pediatric Glaucomas. Philadelphia: Elsevier Inc; 2006:27-39.

15. Quigley HA. The pathogenesis of reversible cupping in congenital glaucoma. Am J Ophthalmol 1977;84:358-70.

16. Quigley HA. Childhood glaucoma: results with trabeculotomy and study of reversible cupping. Ophthalmology 1982;89:219-26.

17. Ely AL, El- Dairi MA, Freedman SF. Cupping reversal in pediatric glaucoma-evaluation of the retinal nerve fiber layer and visual field. Am J Ophthalmol 2014;158:905-15.

18. Raghu N, Pandav SS, Kaushik S, Ichhpujani P, Gupta A. Effect of trabeculectomy on RNFL thickness and optic disc parameters using optical coherence tomography. Eye 2012;26:1131-7.

19. Yanni SE, Wang J, Cheng CS, et al. Normative reference ranges for retinal nerve fiber layer, macula, and retinal layer thicknesses n children. Am J Ophthalmol 2013;155:354-60.

20. Zhu BD, Li SM, Li H, et al. Anyang Childhood Eye Study Group. Retinal nerve fiber layer thickness in a population of 12-year-old children in central China measured by iVue-100 spectral-domain optical coherence tomography: the Anyang Childhood Eye Study. Invest Ophthalmol Vis Sci 2013;54:8104-11.

21. Kang MT, Li SM, Li H, et al. Peripapillary retinal nerve fiber layer thickness and its association with refractive error in Chinese children: the Anyang Childhood Eye Study. Clin Experiment Ophthalmol 2016;44:701-9.

22. Srinivasan S, Addepalli UK, Rao HL, Garudadri CS, Mandal AK. Spectral domain optical coherence tomography in children operated for primary congenital glaucoma. Br J Ophthalmol 2014;98: $162-5$.

23. Zenteno JC, Hernandez-Merino E, Mejia-Lopez H, et al. Contribution of CYP $1 B 1$ mutations and founder effect to primary congenital glaucoma in Mexico. J Glaucoma 2008;17:189-92.

24. Morales- Fernandez L, Martinez-de-la-Casa JM, Garcia-Feijoo J, et al. Clinical variability of primary congenital glaucoma in a Spanish family with Cyp1b1 gene mutations. J Glaucoma 2015;24:630-34. 